\title{
STUNTED PROJECTIVE SPACES AND THE $J$-ORDER OF THE HOPF BUNDLE
}

\author{
BY S. FEDER AND S. GITLER
}

Communicated by William Browder, December 17, 1973

Let $F P^{n}$ be the projective space of dimension $n$ over $F$ where $F=C, Q$ ( $Q$ stands for quaternions). We have the natural cofibrations

$$
F P^{m-1} \rightarrow F P^{n} \rightarrow F P_{m}^{n}
$$

for $m \leqq n$.

Let $H$ denote the Hopf bundle over $F P^{n}$. It is well known that the stunted projective space $F P_{k}^{n+k}$ can be identified with the Thom space of $k H$ over $F P^{n}$, this last being denoted by $\left(F P^{n}\right)^{k H}$.

We study the stable homotopy types of stunted projective spaces $F P_{k}^{n+k}$ for $F=C$ and $F=Q$ and obtain a complete classification. This classification is given in terms of the $J$-order of the Hopf bundle over $F P^{n}$.

We denote by $A_{n}$ the $J$-order of $H$ over $C P^{n}$, and by $B_{n}$ the $J$-order of $H$ over $Q P^{n}$. With this notation the results are:

THEOREM A. The spaces $\left(C P^{n}\right)^{k H}$ and $\left(C P^{n}\right)^{l H}$ are of the same stable homotopy type if and only if one of the following conditions holds: $(n \neq 2,4)^{1}$

(i) $k-l \equiv O\left(A_{n}\right)$,

(ii) $k-l \equiv O\left(A_{n-1}\right)$ and $k+l \equiv O\left(A_{n}\right)$,

(iii) $k-l \equiv O\left(A_{n-1}\right)$ and $k+l+2(n+1) \equiv O\left(A_{n}\right)$.

THEOREM B. The spaces $\left(Q P^{n}\right)^{k H}$ and $\left(Q P^{n}\right)^{l H}$ are of the same stable homotopy type if and only if one of the following conditions holds:

(i) $k-l \equiv O\left(B_{n}\right)$,

(ii) $k-l \equiv O\left(B_{n-1}\right)$ and $k+l \equiv O\left(B_{n}\right)$.

In [1] we have proven that the conditions in Theorem $\mathrm{A}$ are necessary and observed that $A_{n}=A_{n-1}$ for $n$ odd which completed the classification

AMS (MOS) subject classifications (1970). Primary 55D15, 55G25; Secondary 55F50.

1 The cases $n=2,4$ are also solved but the numerical conditions will not be stated here. 
for that case. To complete the classification we construct the necessary homotopy equivalences. We also prove ${ }^{2}$

THEOREM C. The J-order $B_{n}$ of the Hopf bundle over $Q P^{n}$ is given by

$$
v_{2}\left(B_{n}\right)=\max \left\{2 n+1,2 j+v_{2}(j) \mid 1 \leqq j \leqq n\right\}
$$

and

$$
v_{p}\left(B_{n}\right)=\max \left\{j+v_{p}(j) \mid 1 \leqq j \leqq 2 n /(p-1)\right\}
$$

when $p$ is an odd prime.

Here $v_{p}(r)$ is the highest exponent of the prime $p$ which divides $r$.

The proofs will appear elsewhere.

\section{REFERENCE}

1. S. Feder and S. Gitler, Stable homotopy types of stunted complex projective spaces, Proc. Cambridge Philos. Soc. 73 (1973), 431.

Centro de Investigacion y Estudios Avanzados del IPN, Apartado Postal 14-740, Mexico 14, D.F.

${ }^{2}$ This result was announced by F. Sigrist and U. Sutter, Cross-sections of symplectic Stiefel manifolds, Notices Amer. Math. Soc. 19 (1972), A-214, but no proof has been published. 